Supporting Information

for

\title{
Protein-Gated Upconversion Nanoparticle-Embedded Mesoporous Silica Nanovehicles via Diselenide Linkages for Drug Release Tracking in Real Time and Tumor Chemotherapy
}

Hua Yan, ${ }^{\dagger, \dagger}$ Jiangtao Dong, ${ }^{\dagger}$ Xuan Huang, ${ }^{\dagger}$ and Xuezhong Du ${ }^{\dagger, *}$

$\dagger$ Key Laboratory of Mesoscopic Chemistry (Ministry of Education), State Key Laboratory of Coordination Chemistry, and School of Chemistry and Chemical Engineering, Nanjing University, Nanjing 210023, People's Republic of China

* School of Pharmaceutical and Materials Engineering, Taizhou University, Taizhou 318000, Zhejiang Province, People's Republic of China

* Corresponding author

E-mail address: xzdu@nju.edu.cn 

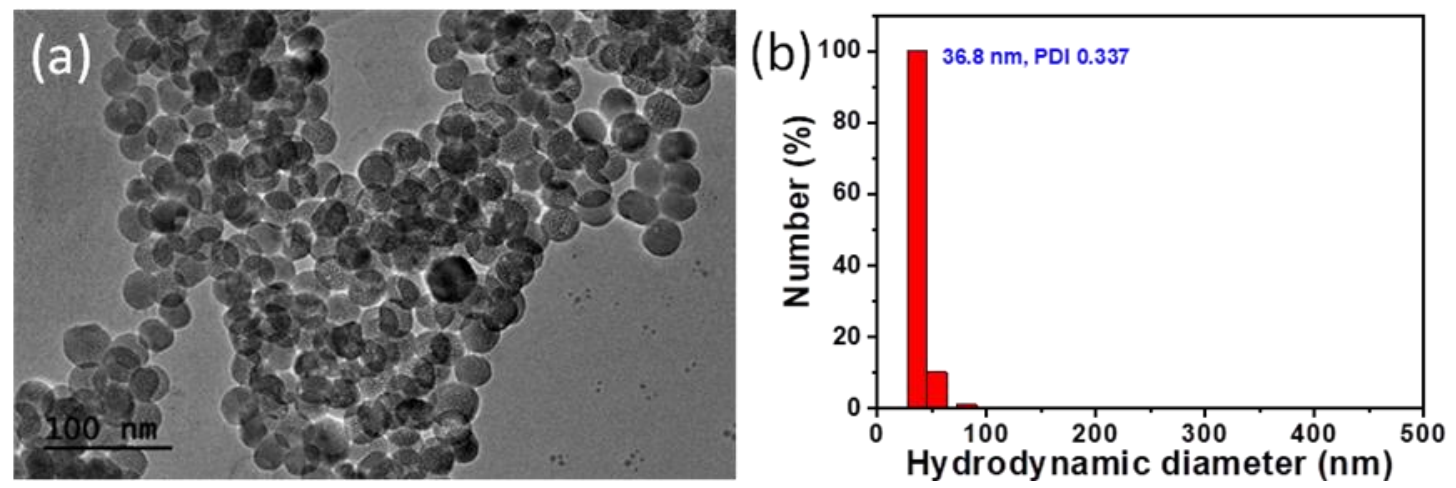

Figure S1. (a) TEM image and (b) hydrodynamic diameters of NaYF4:Yb/Er UCNPs.

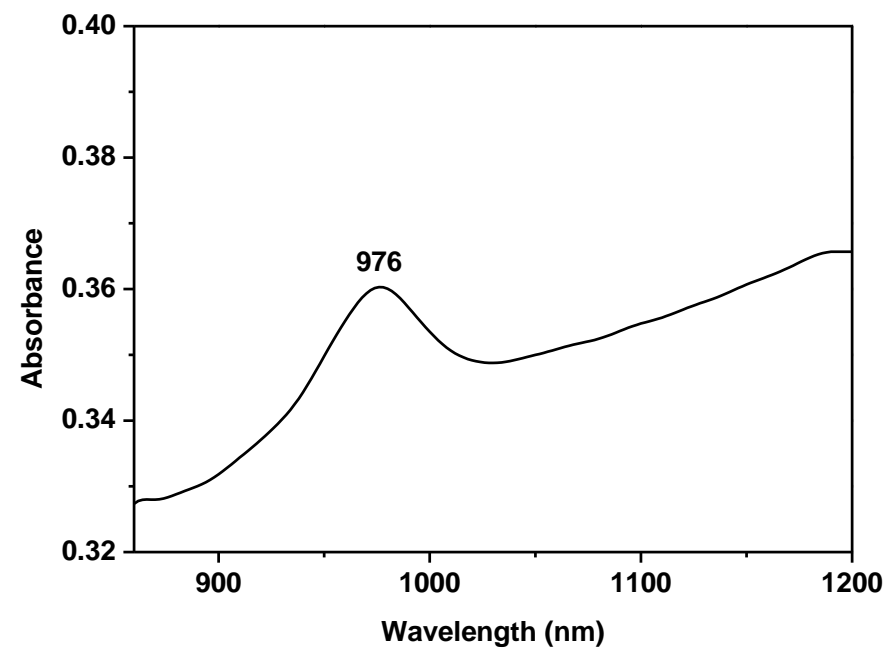

Figure S2. Diffuse reflectance absorption spectrum of UCNP@ $\mathrm{SiO}_{2}$ in the near infrared (NIR) region. 


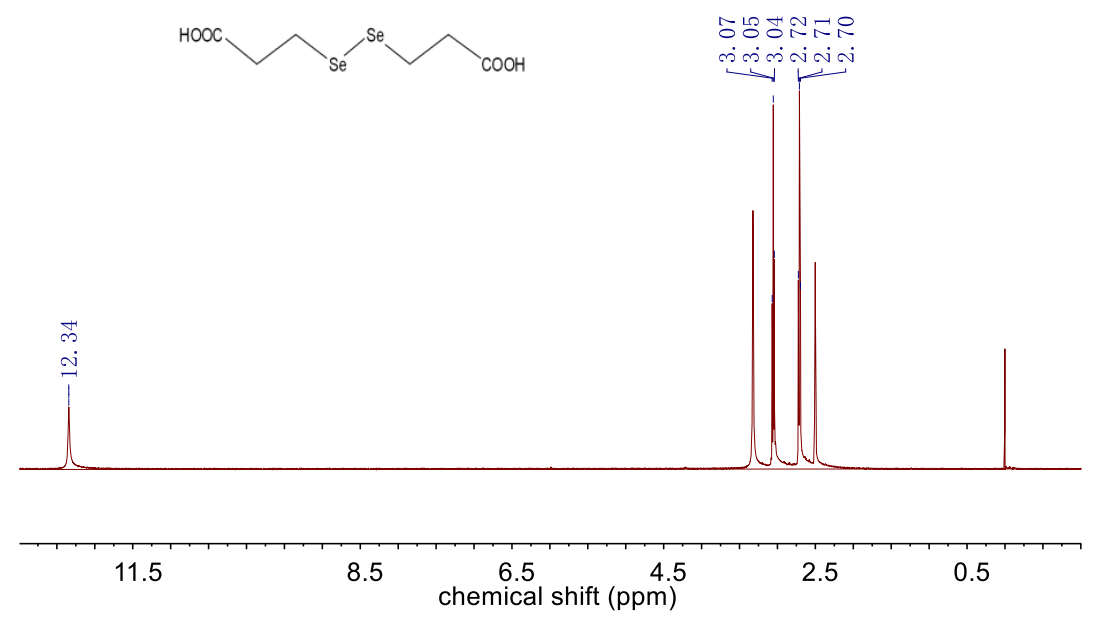

Figure S3. ${ }^{1} \mathrm{H}$ NMR spectrum of DSeDPA in DMSO-d6.

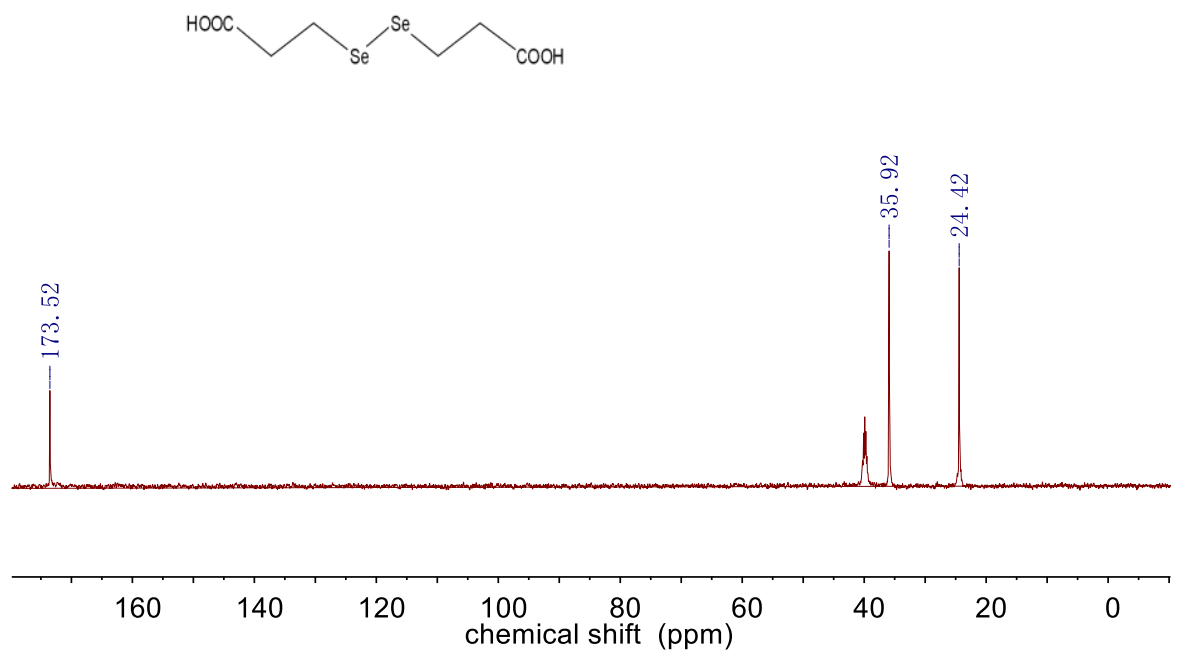

Figure S4. ${ }^{13} \mathrm{C}$ NMR spectrum of DSeDPA in DMSO- $d_{6}$. 

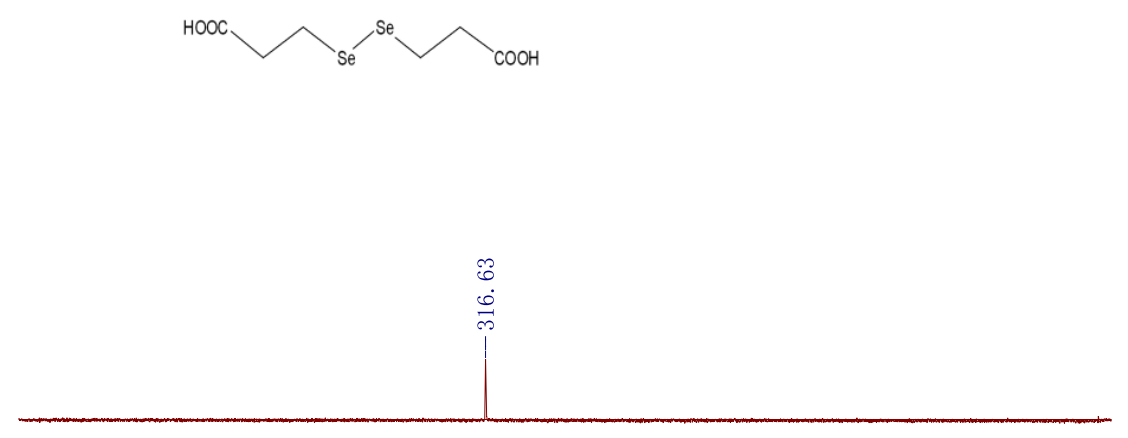

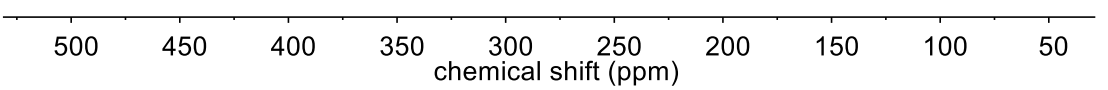

Figure S5. ${ }^{77} \mathrm{Se}$ NMR spectrum of DSeDPA in DMSO- $d_{6}$.

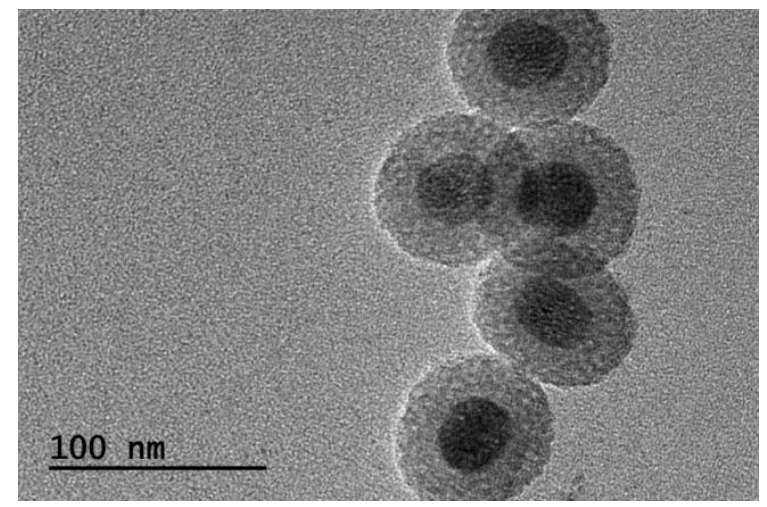

Figure S6. TEM image of UCNP@ $\mathrm{mSiO}_{2}-\mathrm{SeSe}-\mathrm{BSA}$. 

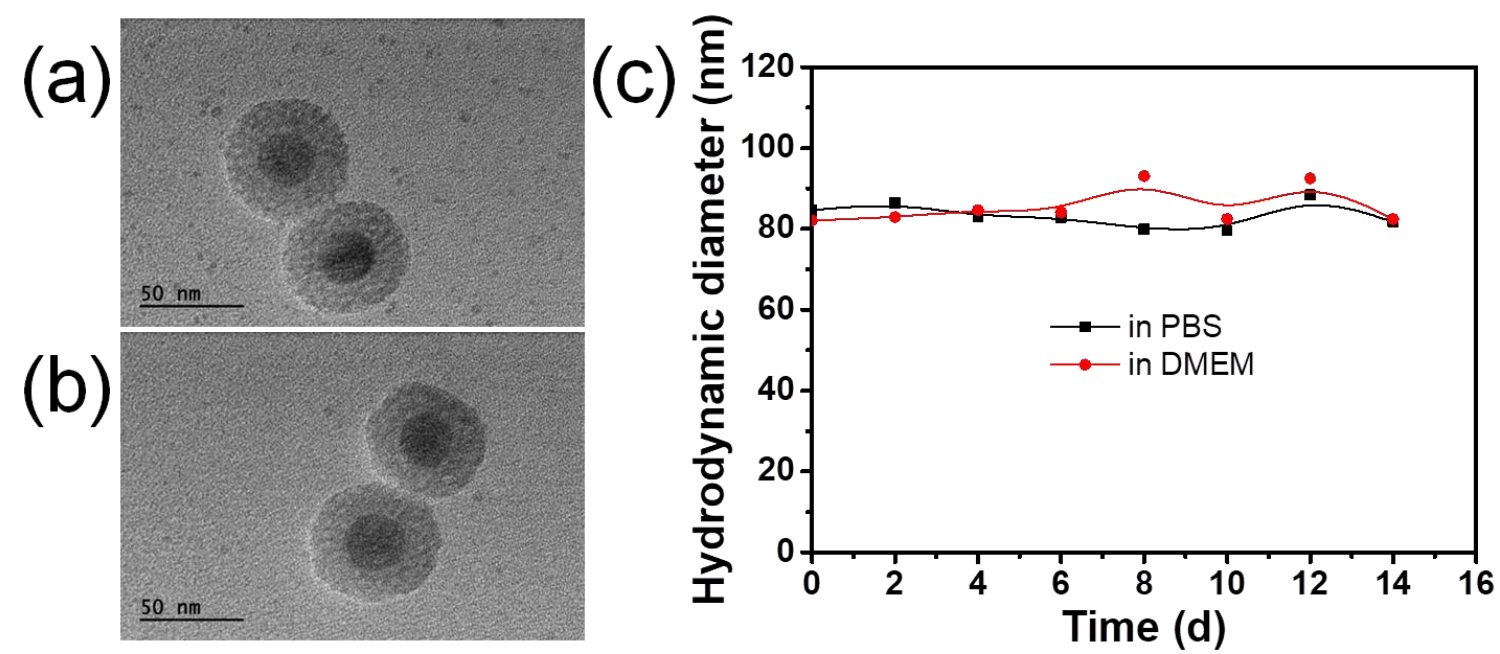

Figure S7. TEM images of UCNP@ $\mathrm{mSiO}_{2}-\mathrm{SeSe}-\mathrm{BSA}$ in (a) phosphate buffered saline (PBS) solution and (b) culture medium (DMEM) after 14 days of storage and (c) hydrodynamic diameters of UCNP@ $\mathrm{mSiO}_{2}-\mathrm{SeSe}-\mathrm{BSA}$ in PBS solution and DMEM during the period of 14 days. 


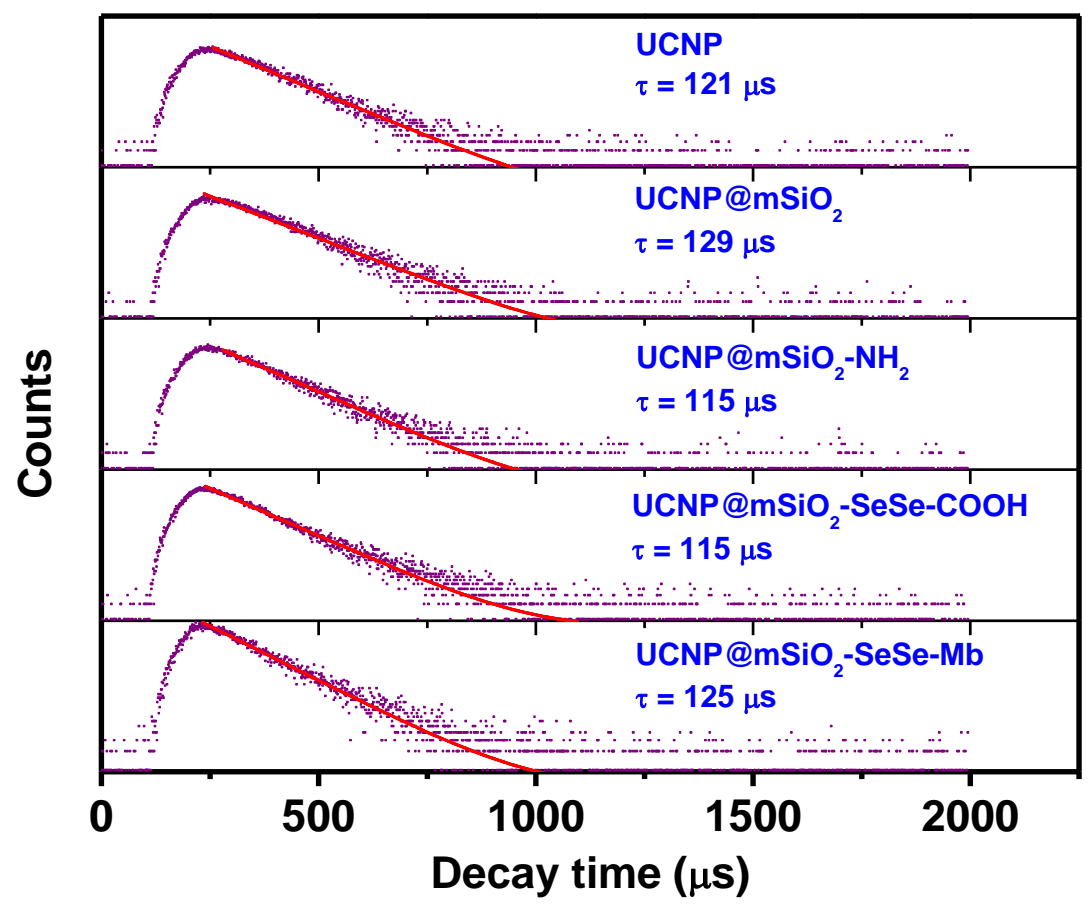

Figure S8. Luminescence decay curves of UCNP, UCNP@ $\mathrm{mSiO}_{2}, \mathrm{UCNP} @ \mathrm{mSiO}_{2}-\mathrm{NH}_{2}$, UCNP@mSiO $2-\mathrm{SeSe}-\mathrm{COOH}$, and UCNP@ mSiO $2-\mathrm{SeSe}-\mathrm{Mb}$ at 543 nm upon excitation of 980 nm. 


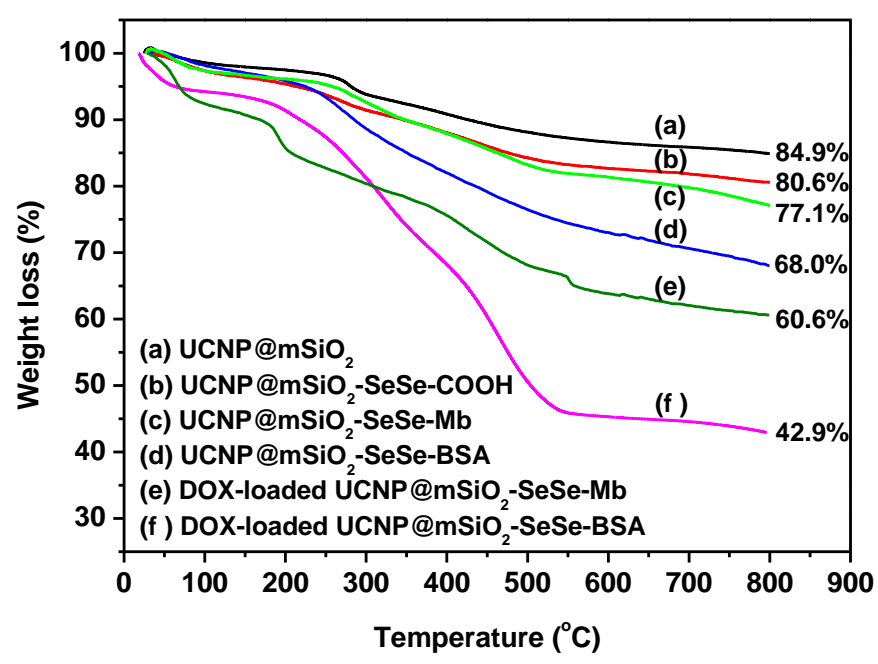

Figure S9. Thermogravimetric analysis profiles of UCNP@ $\mathrm{mSiO}_{2}, \mathrm{UCNP} @ \mathrm{mSiO}_{2}-\mathrm{SeSe}-$ COOH, UCNP@ mSiO2-SeSe-Mb, UCNP@ mSiO 2-SeSe-BSA, DOX-loaded UCNP@mSiO2SeSe-Mb, and DOX-loaded UCNP@ mSiO2-SeSe-BSA. 

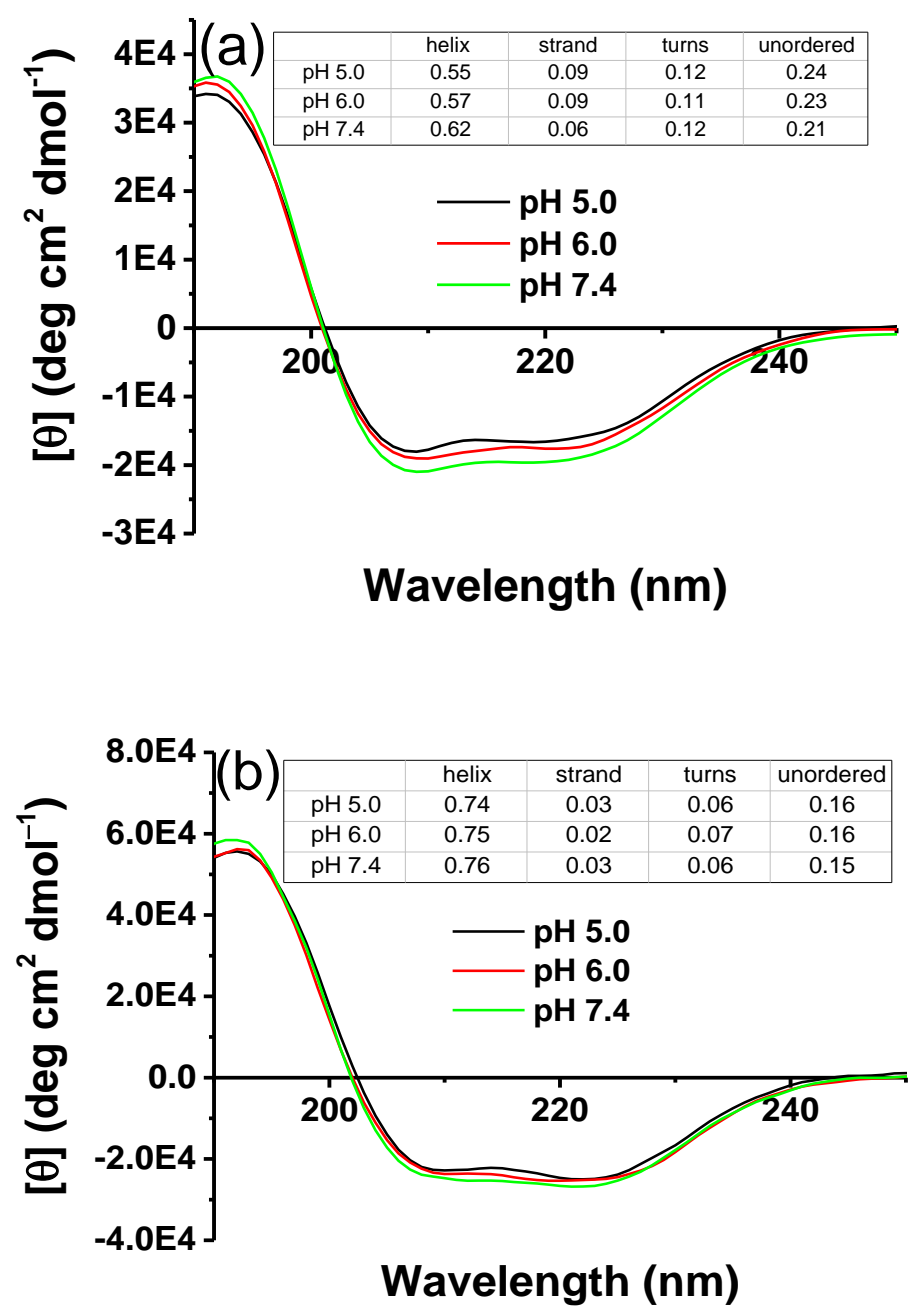

Figure S10. CD spectra of aqueous solutions of (a) BSA and (b) Mb at different $\mathrm{pH}$ values.

The analyses of protein secondary structures were calculated using the Dichroweb webserver. ${ }^{\mathrm{S} 1}$ 

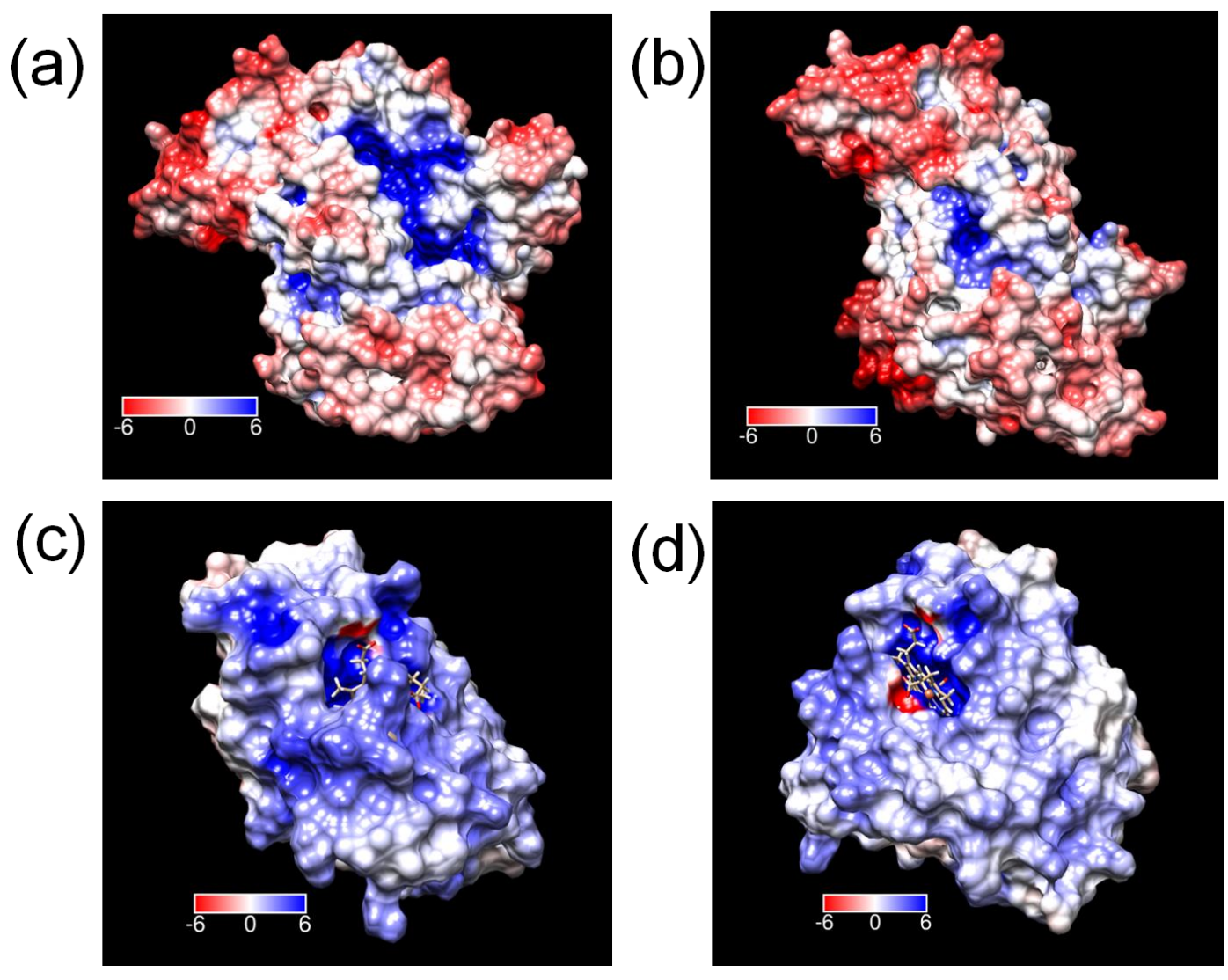

Figure S11. Electrostatic potential maps of the protein surfaces with different amino acid residues: (a,b) BSA; (c,d) Mb. The crystal structures of BSA (PDB ID: 4f5s) and Mb (PDB ID: 1dwr) were obtained from the Protein Data Bank (PDB), and visualization and analyses of structures and electrostatic potential maps of BSA and Mb were calculated.

The visualization and analyses of structures and electrostatic potential maps of BSA and Mb were calculated by us using the procedure UCSF Chimera. ${ }^{\mathrm{S}}$ 


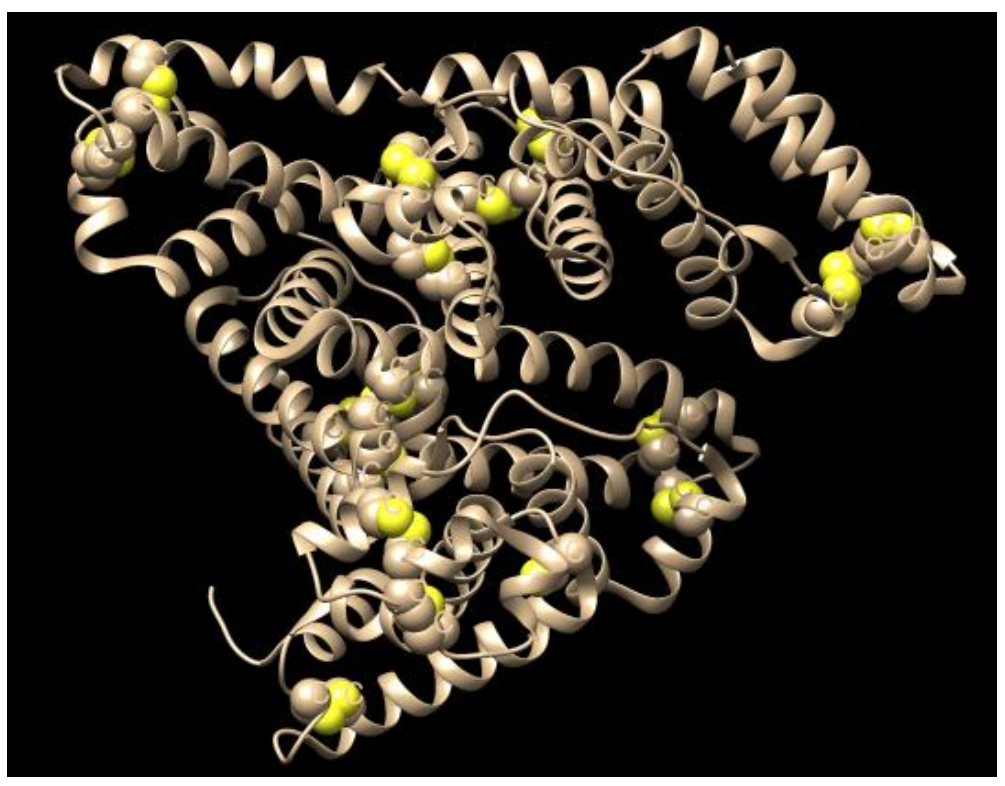

Figure S12. Distribution of $17 \mathrm{~S}-\mathrm{S}$ bridges and one sulfhydryl group in the structure of BSA. The crystal structure of BSA (PDB ID: 4f5s) was obtained from the Protein Data Bank (PDB). 


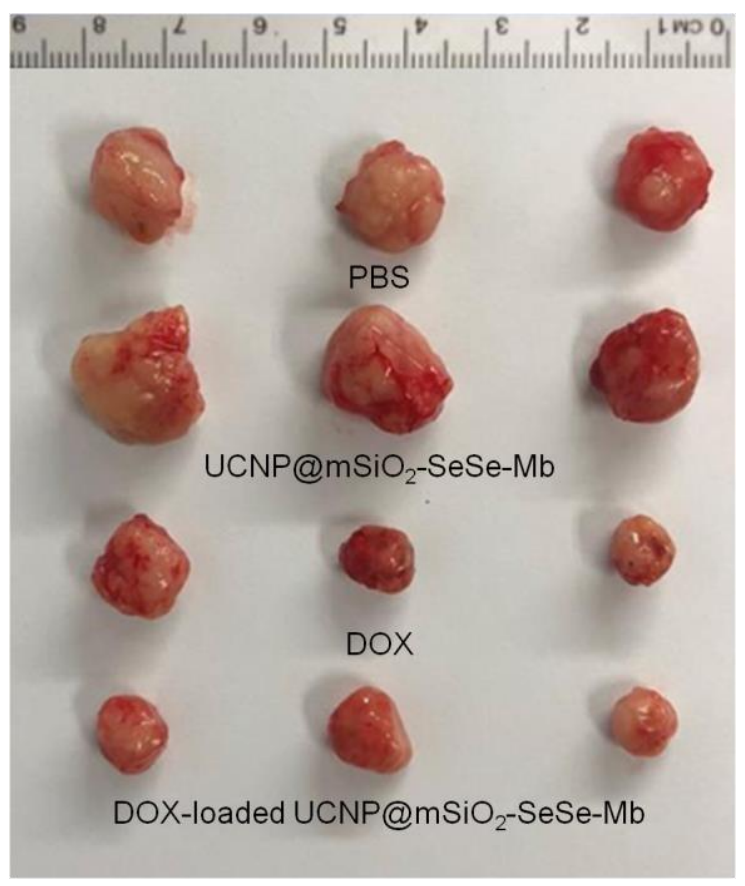

Figure S13. Photographs of the tumors collected from different groups of treated mice after 27 days $(n=3)$.

Table S1. Weights of the tumors collected from different groups of treated mice after 27 days $(n$ $=3$ ).

\begin{tabular}{ll}
\hline group of treated mice & tumor weight $[\mathrm{g}]$ \\
\hline PBS & $0.7637 \pm 0.1178$ \\
$\mathrm{UCNP} @ \mathrm{mSiO}_{2}-\mathrm{SeSe}-\mathrm{Mb}$ & $1.3787 \pm 0.4311$ \\
bare DOX & $0.3900 \pm 0.1750$ \\
DOX-loaded UCNP@mSiO $2-S e S e-M b$ & $0.3520 \pm 0.1367$
\end{tabular}




\section{References}

(S1) Whitmore, L.; Wallace, B.A. Protein Secondary Structure Analyses from Circular Dichroism Spectroscopy: Methods and Reference Databases. Biopolymers, 2008, 89, $392-400$.

(S2) Pettersen, E. F.; Goddard, T. D.; Huang, C. C.; Couch, G. S.; Greenblatt, D. M.; Meng, E. C.; Ferrin, T. E. UCSF Chimera-A Visualization System for Exploratory Research and Analysis. J. Comput. Chem. 2004, 25, 1605-1612. 